\title{
A Taxonomy for Selecting Wearable Input Devices for Mixed Reality
}

Ahmed S. Khalaf

Play \& Interactive Experiences

for Learning Lab

Computer Science Department

New Mexico State University

khalaf@nmsu.edu

\section{Sultan A. Alharthi}

Play \& Interactive Experiences

for Learning Lab

Computer Science Department

New Mexico State University

salharthi@nmsu.edu

Son Tran

Computer Science Department

New Mexico State University

stran@nmsu.edu
Igor Dolgov

Psychology Department

Computer Science Department

New Mexico State University

id@nmsu.edu

\section{Z O. Toups}

Play \& Interactive Experiences for Learning Lab

Computer Science Department New Mexico State University z@cs.nmsu.edu

Permission to make digital or hard copies of part or all of this work for personal or classroom use is granted without fee provided that copies are not made or distributed for profit or commercial advantage and that copies bear this notice and the full citation on the first page. Copyrights for third-party components of this work must be honored. For all other uses, contact the owner/author(s).

ISS'19, November 10-13, 2019, Daejeon, Republic of Korea

Copyright is held by the author/owner(s).

ACM ISBN 978-1-4503-6891-9/19/11.

http://dx.doi.org/10.1145/3343055.3360759

\begin{abstract}
Composite wearable computers consist of multiple wearable devices connected together and working as a cohesive whole. These composite wearable computers are promising for augmenting our interaction with the physical, virtual, and mixed play spaces (e.g., mixed reality games). Yet little research has directly addressed how mixed reality system designers can select wearable input devices and how these devices can be assembled together to form a cohesive wearable computer. We present an initial taxonomy of wearable input devices to aid designers in deciding which devices to select and assemble together to support different mixed reality systems. We undertook a grounded theory analysis of 84 different wearable input devices resulting in a design taxonomy for composite wearable computers. The taxonomy consists of two axes: TYPE OF INTERACTIVITY and BODY LOCATION. These axes enable designers to identify which devices fill particular needs in the system development process and how these devices can be assembled together to form a cohesive wearable computer.
\end{abstract}

\section{Author Keywords}

Wearable input devices; wearable devices; wearable technology; wearable taxonomy 


\section{Introduction \& Background}

Recent advances in mobile and ubiquitous technologies have opened countless possibilities for the growth of wearable computers, which are often purpose-built individual devices $[8,13,19]$. The present research is concerned with composite wearable computers - a combination of multiple wearables that are integrated together for a particular purpose and that must be assembled from multiple technologies. Composite wearable computers generally represent a space for research, aiming to support new use cases in existing activities and which could then lead to more commercial devices. Examples of composite wearable computers from the literature include $[3,11,16,17]$. The present research contributes an initial taxonomy of wearable input devices to aid designers in deciding on which devices to assemble for mixed reality systems [12]

Wearable devices have become a part of everyday life for many, as they enhance individuals' security and comfort. One of the main characteristics of wearable computers is the ability to integrate into daily activities, often functioning as a secondary interface to support a wearer's primary task, requiring minimal attention [2].Prior research has proposed classifications of wearable based on application $[1,14]$ or identify design principles for wearable user interfaces that support ergonomics and interface design $[5,10,19]$. Yet little research has addressed how mixed reality system designers can select wearable input devices and how these devices can be assembled together to form a cohesive wearable computer.

Systems that connect virtual and physical reality in some meaningful way through the use of networks, sensors, and databases are mixed realities [9]. These range from augmented reality, in which conformal 3D imagery is integrated with a perspective on the physical world to augmented vir- tuality, in which physical-world artifacts and spaces are integrated into a virtual world [12].

Wearables offer a range of input modalities, including speech commands, touch screens and pads, air-based gestures,

hand-signals, movement tracking, and various forms of context capture [4], which maximize usability and mobility. For example, some wearable devices allow for hands-free interaction through the use of speech interfaces and voice-totext conversion (e.g., Sony SmartEyeglass [15]). Alternatively, several wearable devices use gestural interaction as an input method; some of these devices allow finger-based and hand-based gesture interactions [7], while others rely on clicking buttons and manual entry of commands [18]. In the present research, we focus on wearable input devices and investigate the best way they can be selected and composed for wearable computing systems. When carefully selected, these input devices have the potential to offer substantial performance improvements in a number of fields without impairing human mobility.

In this work, we focus on input devices; feedback devices (e.g., displays, haptics) are beyond the scope of the current work. The purposed taxonomy is intended to guide mixed reality system designers through the process of selecting a wearable device or a set of wearable devices upon which they can build their systems. Based on these motivations we posed the following research questions:

RQ1: What range of wearable input devices are presently available?

RQ2: What capabilities do these existing wearable input devices have?

RQ3: What devices are compatible with each other on the body of a single user? 


\section{Source Description}

$P N N L$ out of nine issues of Responder Technology Alert journal (20152016), we found 36 wearable input devices.

ACM out of 37 studies

(CHI) from $\mathrm{CHI}$ conference (1997-2017), we found 15 wearable input devices

ACM out of 18 studies

(Ubi- from UbiComp

Comp) conference (20142016), we found 7 devices

IEEE out of 15 studies

(ISWC) from ISWC conference (1997-2012), we found 9 devices

Others 17 devices were selected based on our prior knowledge of wearables, or was found while looking for data for other wearable devices.

Table 1: Sources of selected wearable input devices
To answer these questions, we analyze wearable devices, their documentation, and prior user studies using them, to create a prescriptive taxonomy for selecting wearable input devices for mixed reality systems using a grounded theory approach

\section{Grounded Theory Methodology}

We conduct a qualitative study of wearable input devices to identify the characteristics of each type and propose a taxonomy to guide the selection and composition of such devices. In this work, we employed a grounded theory approach [6], which is a set of research practices for exploring and characterizing a new domain [6].

\section{Process Overview}

We started with an iterative process of finding and selecting wearable input devices and collecting data from multiple sources for each wearable device; then performing open coding to identify the initial concepts, categories, and their features; then gradually building these up into a set of axial codes that form a taxonomy.

We started with searching for and selecting wearable input devices from three different sources: Pacific Northwest $\mathrm{Na}$ tional Laboratory (PNNL) reports ${ }^{1}$, the ACM Digital Library, ${ }^{2}$, and IEEE Xplore Digital Library, ${ }^{3}$ (see table 1 ), followed by collecting data on each of the discovered wearable devices. Our focus is on devices that enable the user to input data directly, not those that sample activities, because our interest is in how people build mixed reality systems.

Selecting Wearable Input Devices

To find existing wearable input devices, addressing RQ1, we searched for specialized journals and conferences that

\footnotetext{
Pacific Northwest National Laboratory: http://www.pnnl.gov

${ }^{2}$ ACM Digital Library: http://dl.acm.org
}

${ }^{3}$ IEEE Xplore Digital Library: http://ieeexplore.ieee.org focus on wearable devices and sought out research papers on the subject ((see Table 1). The resulting search from these different journals and conferences identified 70 total research papers. Also, we added 17 wearable input devices to our list. These devices are selected based on our prior knowledge of wearables, or was found while looking for data for other wearable devices. By the end of this process, we identified 84 unique wearable input devices to include in the analysis.

\section{Analysis Procedure}

Our analysis involved several phases which served to characterize the device space and address RQ2 and RQ3:

Phase 1: Initial Observations of Device Features The primary goal of this phase was to identify the capabilities of each device, its range of expressiveness, and what it might be used for. For each device, we identified the technologies with which it was constructed; the form factor of the device and where it is worn; and the application domain of the device.

Phase 2: Open Coding on Collected Data

In this step, we began developing codes to classify devices. Two researchers participated in this process, independently reading through the device network in ATLAS.ti and identifying keywords in device descriptions and data sources.

\section{Phase 3: Axial and Selective Coding}

During this phase, we engaged in multiple iterative discussion sessions to explore the relationship between the codes, the emergent concepts, and the initial categories.

All these phases together resulted in a taxonomy for selecting wearable input devices for mixed reality systems. In the following, we provide an overview of the taxonomy and its axes, and discuss future work 


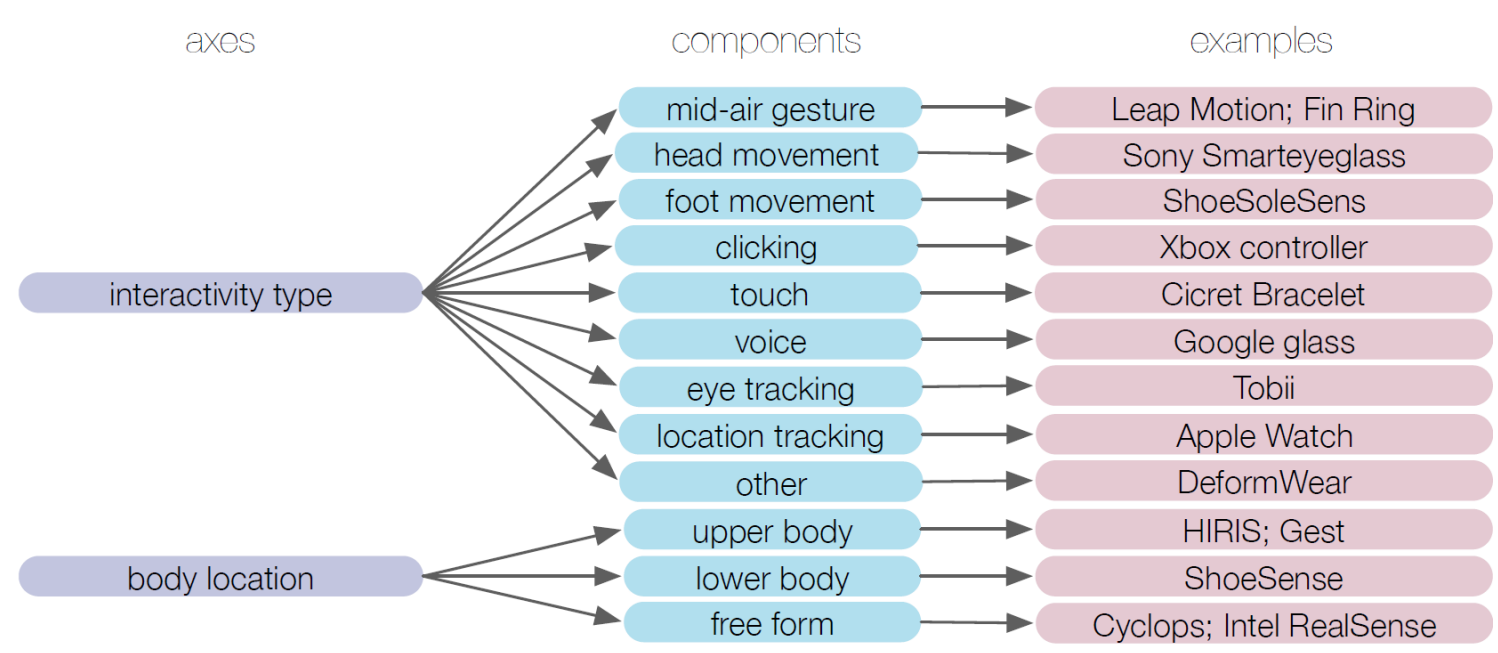

Figure 1: A thematic tree describing the taxonomy to support composite wearable computers.

\section{Taxonomy Overview}

Our taxonomy of wearable input devices contains two axes: TYPE OF INTERACTIVITY, and BODY LOCATION (Figure 1). The axes provide significant information about each wearable device, including: what type of interactivity the device allows, and where the device can be worn. This information about each device then provides designers with the needed details to build composite wearable computers.

\section{TYPE OF INTERACTIVITY AXis}

We define the interactivity axis as the input modality or modalities through which the user expresses their intentions using the wearable device. TYPE OF INTERACTIVITY considers how a user may invoke actions with a device (e.g., voice, gesture interaction, clicking, touching). For the TYPE OF INTERACTIVITY axis a device may have multiple modal- ities (e.g., a device that enables both voice interaction and gesture interaction) and so may exist at multiple points on the axis. We expect that as developers consider composing together devices they will identify which types of interactivity they need to support in their applications. They can then consult the taxonomy to identify which device, or combination of devices, is necessary.

\section{BODY LOCATION Axis}

Wearable devices come in many forms and fit different parts of the body: for example, devices worn on the head (glasses), hand(s) (gloves or rings), and wrist(s) (watches). While we expect users to equip multiple wearables for a single composite wearable computer, certain combinations are not comfortable or not possible (e.g., wearing a ring and a glove on the same hand). The BODY LOCATION axis ad- 
dresses the position of the body on which the device may be worn, enabling the designer to identify which combinations of devices can be composed together (and which might be mutually exclusive). Again, devices may cover multiple positions on the axis.

All of these different factors that may influence how a wearable device can be selected show how such selection poses significant challenges to designers. The purposed taxonomy provides a palette for mixed reality system designers to select wearable devices and a starting point for understanding how these devices can be assembled together to form a cohesive wearable computer.

\section{Conclusion \& Future Work}

This work-in-progress presents an initial taxonomy for selecting wearable input devices for mixed reality systems. We expect our complete taxonomy to be of value to mixed reality system designers and researchers, and hope that, through this work, we discover new ways for selecting and assembling wearable computers that can support different mixed reality systems and experiences.

For future work, we will continue improving and refining the taxonomy. Extending the taxonomy to also include output devices. Also, we will validate the taxonomy through designing different composed wearable computer for mixed reality systems, and investigative different aspects of the wearable taxonomy through future user studies with designers and players.

\section{Acknowledgments}

This material is based upon work supported by the National Science Foundation under Grant Nos. IIS-1651532 and IIS1619273.

\section{REFERENCES}

1. Mayda Alrige and Samir Chatterjee. 2015. Toward a taxonomy of wearable technologies in healthcare. In International Conference on Design Science Research in Information Systems. Springer, 496-504.

2. Woodrow Barfield. 2015. Fundamentals of wearable computers and augmented reality. CRC Press.

3. M. Boronowsky, T. Nicolai, C. Schlieder, and A. Schmidt. 2001. Winspect: a case study for wearable computing-supported inspection tasks. In Proceedings Fifth International Symposium on Wearable Computers. 163-164. DOI : http://dx.doi.org/10.1109/ISWC. 2001.962124

4. Paul Dourish. 2004. What we talk about when we talk about context. Personal and Ubiquitous Computing 8, 1 (01 Feb 2004), 19-30. DOI :

http://dx.doi.org/10.1007/s00779-003-0253-8

5. F. Gemperle, C. Kasabach, J. Stivoric, M. Bauer, and R. Martin. 1998. Design for wearability. In Digest of Papers. Second International Symposium on Wearable Computers (Cat. No.98EX215). 116-122. DOI : http://dx.doi.org/10.1109/ISWC.1998.729537

6. Barney G. Glaser and Anselm Strauss. 1967. The Discovery of Grounded Theory: Strategies for Qualitative Research. Aldine Publishing Company, Chicago, Illinois, USA.

7. Yasuyuki Kono and Yuki Tanaka. 2016. A Wearable Handwriting System for Time-warping Collocation. In Proceedings of the 18th International Conference on Human-Computer Interaction with Mobile Devices and Services Adjunct (MobileHCl '16). ACM, New York, NY, USA, 1121-1124. DOI :

http://dx.doi.org/10.1145/2957265.2962652 
8. Steve Mann. 1998. Wearable computing as means for personal empowerment. In Proc. 3rd Int. Conf. on Wearable Computing (ICWC). 51-59.

9. Paul Milgram and Fumio Kishino. 1994. A taxonomy of mixed reality visual displays. IEICE Trans. Information Systems E77-D, 12 (1994), 1321-1329.

10. Vivian Motti and Kelly Caine. 2014. Human factors considerations in the design of wearable devices. In Proceedings of the Human Factors and Ergonomics Society Annual Meeting, Vol. 58. SAGE Publications Sage CA: Los Angeles, CA, 1820-1824.

11. Tom Nicolai, Thomas Sindt, Hendrik Witt, Jörn Reimerdes, and Holger Kenn. 2006. Wearable computing for aircraft maintenance: Simplifying the user interface. In 3rd International Forum on Applied Wearable Computing 2006. VDE, 1-12.

12. Hitesh Nidhi Sharma, Sultan A. Alharthi, Igor Dolgov, and Zachary O. Toups. 2017. A Framework Supporting Selecting Space to Make Place in Spatial Mixed Reality Play. In Proceedings of the Annual Symposium on Computer-Human Interaction in Play (CHI PLAY '17). ACM, New York, NY, USA, 83-100. DOI : http://dx.doi.org/10.1145/3116595.3116612

13. Hitesh Nidhi Sharma, Zachary O. Toups, Igor Dolgov, Andruid Kerne, and Ajit Jain. 2016. Evaluating Display Modalities Using a Mixed Reality Game. In Proceedings of the 2016 Annual Symposium on Computer-Human Interaction in Play (CHI PLAY'16). ACM, New York, NY, USA, 65-77. DOI : http://dx.doi.org/10.1145/2967934.2968090

14. Roy Shilkrot, Jochen Huber, Jürgen Steimle, Suranga Nanayakkara, and Pattie Maes. 2015. Digital Digits: A Comprehensive Survey of Finger Augmentation
Devices. ACM Comput. Surv. 48, 2, Article 30 (Nov. 2015), 29 pages.

15. Sony. 2015. Sony Releases the Transparent Lens Eyewear "SmartEyeglass Developer Edition". https://www. sony. net/SonyInfo/News/Press/ 201502/15-016E/index.html. (17 March 2015).

16. Bruce Thomas, Victor Demczuk, Wayne Piekarski, David Hepworth, and Bernard Gunther. 1998. A wearable computer system with augmented reality to support terrestrial navigation. In Digest of Papers. Second International Symposium on Wearable Computers (Cat. No. 98EX215). IEEE, 168-171.

17. Hendrik Witt and Ernesto Morales Kluge. 2008. Studying input device performance: an end-user driven experiment in wearlT@ work. In First International Conference on Advances in Computer-Human Interaction. IEEE, 9-15.

18. Robert Xiao, Gierad Laput, and Chris Harrison. 2014. Expanding the Input Expressivity of Smartwatches with Mechanical Pan, Twist, Tilt and Click. In Proceedings of the SIGCHI Conference on Human Factors in Computing Systems (CHI '14). ACM, New York, NY, USA, 193-196. DOI :

http://dx.doi.org/10.1145/2556288.2557017

19. Clint Zeagler. 2017. Where to Wear It: Functional, Technical, and Social Considerations in On-body Location for Wearable Technology 20 Years of Designing for Wearability. In Proceedings of the 2017 ACM International Symposium on Wearable Computers (ISWC '17). ACM, New York, NY, USA, 150-157. DOI : http://dx.doi.org/10.1145/3123021.3123042 